Three-Dimensional Finite Element Analysis of Spatially Variable PVD Improved-Ground

Md. Wasiul Bari*

Department of Civil Engineering, Curtin University, WA 6845, Australia

E-mail: $\underline{\text { Md.Bari@curtin.edu.au }}$

Mohamed A. Shahin

Department of Civil Engineering, Curtin University, WA 6845, Australia

E-mail: M.Shahin@curtin.edu.au

*Corresponding author 


\title{
Three-Dimensional Finite Element Analysis of Spatially Variable PVD Improved-Ground
}

\begin{abstract}
A stochastic approach that investigates the effects of soil spatial variability on stabilization of soft clay via prefabricated vertical drains (PVDs) is presented and discussed. The approach integrates the local average subdivision of random field theory with the Monte Carlo finite element technique. A special feature of the current study is the investigation of impact of spatial variability of soil permeability and volume compressibility in the smear zone as compared to that of the undisturbed zone, in conjunction with uncoupled 3D finite element analysis. A sensitivity analysis is also performed to identify the random variable that has the major contribution to the uncertainty of the degree of consolidation achieved via PVDs. The results of this study indicate that the spatial variability of soil properties has a significant impact on soil consolidation by PVDs; however, the spatial variability of soil properties in the smear zone has a dominating impact on soil consolidation by PVDs over that of the undisturbed zone. It is also found that soil volume compressibility has insignificant contribution to the degree of consolidation estimated by uncoupled stochastic analysis.
\end{abstract}

Keywords: soil consolidation; prefabricated vertical drains; finite element method; Monte Carlo technique; soil spatial variability.

\section{Introduction}

Soils are highly variable from one point to another in the ground. This inherent variation of soils with respect to spatial location is known as soil spatial variability and is due to the uneven soil micro fabric, complex characteristics of geological deposition and stress history. Despite the fact that the impact of spatial variation of soil properties on soil consolidation has long been recognized by many researchers (e.g. Pyrah 1996; Rowe 1972), the design of soil consolidation via prefabricated vertical drains (PVDs) has been traditionally carried out deterministically and thus can be misleading due to the ignorance of the uncertainty associated with the inherent spatial variation of soil properties. In general, acknowledging and quantifying the soil spatial variability in 
geotechnical engineering has been usually considered using probabilistic modelling techniques that treat the soil properties as random variables resulting in more realistic solutions. Unlike deterministic analyses, which are based on single best estimate (average or characteristic) values of soil properties, the probabilistic analyses explicitly take into account the variable nature of soil properties, based on their statistical characteristics.

The formulation and solution of stochastic problems are often very complicated. The review of relevant literature has indicated that although the significance of soil spatial variability in relation to ground improvement by PVDs has long been realized, little research has been made in this area. Given the analytical and numerical complexity of the problem, available research into the consolidation of highly variable soils has been limited to the following two categories: (i) one-dimensional consolidation due to vertical drainage, i.e. no PVDs, for either 1D or 2D geometries (e.g. Badaoui et al. 2007; Freeze 1977; Houmadi et al. 2012; Huang et al. 2010; Hwang and Witczak 1984); and (ii) soil consolidation by PVDs considering only the uncertainty associated with the measurement errors of soil testing, while the inherent spatial variability of soil properties has not been taken into account and soil permeability, $k$, and coefficient of volume compressibility, $m_{v}$, are combined into a single coefficient of consolidation (e.g. Hong and Shang 1998; Zhou et al. 1999). However, it has been suggested by many researchers (e.g. Huang and Griffiths 2010; Huang et al. 2010; Lee et al. 1992; Pyrah 1996) that the use of $k$ and $m_{v}$ as independent parameters is a better choice for the numerical analyses because the coefficient of consolidation cannot explicitly account for the true combined effects of $k$ and $m_{v}$, and usually yields incorrect pore pressure distributions. More recently, preliminary studies have been carried out by the authors (e.g. Bari et al. 2012; 2013; Shahin and Bari 2012) on soil spatial variability for 
consolidation of soft clays by PVDs and have shown valuable insights into the impact of soil spatial variability on soil consolidation and enhanced conceptual understanding about the soil consolidation problem. However, the above mentioned works have notable limitations of either ignoring the smear effect or considering smear effect with reference to permeability changes alone and volume compressibility have been ignored. It has to be noted that, the smear effect that develops as a consequence of mandrel installation not only reduces $k$ but also increases $m_{v}$. The combined effect of reduced $k$ and increased $m_{v}$ within the smear zone brings different behaviour from that of the undisturbed soil. Hence, for more accurate prediction of the behaviour of stabilized soil with PVDs, the changes of both $k$ and $m_{v}$ in the smear zone as well as undisturbed zone need to be considered. However, due to the non-uniform spatial distribution of soil disturbance (which decreases with the increase of distance from the centre of the drain), the variability characteristics of the smeared soil may be significantly different from those of undisturbed soil. In addition, as expelled water must pass through the smear zone, the implication of variability parameters in this zone on the overall consolidation behaviour may be different from that of the variability parameters in the undisturbed zone. Furthermore, in case of multiple spatially variable parameters, the effect of one soil property on the estimated behaviour of soil consolidation may be relatively more significant than that of another soil property even with the same magnitude of spatial variation. Therefore, it is necessary to distinguish the spatially variable soil parameters that have the dominating influence on the system response as this leads to a potential reduction in the number of spatially variable parameters that need to be considered in the analysis. In this paper, a parametric study is carried out to investigate the relative significance of spatially variable soil properties in the smear zone over the undisturbed zone, where $k$ and $m_{v}$ are individually treated as random variables. A sensitivity analysis 
is also performed to identify the random variable that has the most significant contribution in the uncertainty of the degree of consolidation achieved via PVDs.

\section{Stochastic modelling of soil consolidation by PVDs}

Among several methods of modelling stochastic problems, the use of deterministic finite element analysis with random input soil parameters in a Monte Carlo framework has gained much popularity in recent years (Elkateb et al. 2003). Similar approach is adopted in the present work to investigate the effects of soil spatial variability on the behaviour of soil consolidation by PVDs. The approach merges the local average subdivision (LAS) method (to generate random permeability fields) and finite element modelling (to calculate soil consolidation by PVDs) into a Monte Carlo framework. For a certain problem of ground improvement by PVDs, the proposed approach can be applied using the following steps:

(1) Create a virtual soil profile for the problem in hand which comprises a grid of elements that is assigned random values of soil properties different from one element to another across the grid. The virtual soil profile allows arbitrary distributions of soil properties to be realistically and economically modelled according to their statistical characteristics;

(2) Incorporate the generated soil profile into a finite element modelling scheme of soil consolidation by PVDs; and

(3) Repeat Steps 1 and 2 many times using the Monte Carlo technique so that a series of consolidation responses can be obtained from which the statistical distribution parameters and probability of achieving a target degree of consolidation can be estimated and analysed.

Details of the steps used, as well as the numerical procedures, are described below. 


\section{Generation of virtual soil profile}

As mentioned earlier, $k$ and $m_{v}$ are considered to be the random variables in the present study and are characterized in terms of their probability density function (PDF) i.e. the mean, $\mu$, standard deviation, $\sigma$ (the standard deviation can also be represented by variance, $\sigma^{2}$, or coefficient of variation, $v$, where, $v=\sigma / \mu$ ). While soil properties vary randomly in the ground, such variation is gradual and spatial dependency exists (Fenton and Vanmarcke 1990; Jaksa et al. 1997; Vanmarcke 1977). That is, a soil property at two separate spatial locations could be similar or otherwise, depending on the distance they are located apart and this is known as spatial correlation. Vanmarcke (1977) pointed out that adequate characterization of spatially variable soil properties requires consideration of such spatial correlation. The mean and standard deviation are the point statistical measures with no consideration of the spatial correlation structure of soil properties. Therefore, a third parameter (i.e. the scale of fluctuation, SOF) is usually introduced as an additional statistic to consider the spatial correlation of soil properties. The SOF is also known as the correlation length and is usually denoted as $\theta$. Generally speaking, a large value of $\theta$ indicates smooth spatial variation of soil property of interest, whereas a small value of $\theta$ implies erratic variation. In this study, the variability of both $k$ and $m_{v}$ is characterized by following a lognormal distribution and assumed as 3D random fields. In selecting the probability distribution of $k$ and $m_{v}$, the authors reviewed a broad range of literature (e.g. Badaoui et al. 2007; Freeze 1977; Huang et al. 2010) and concluded that it is reasonable to assume lognormal probability distribution for both $k$ and $m_{v}$. Since the same approach is used to generate random field of both $k$ and $m_{v}$, only the procedure to generate the random field of $k$ is summarized herein. In the process of simulating the lognormally distributed random field of $k$, correlated local averages standard normal random field $G(x)$ are first generated with zero mean, 
unit variance and a spatial correlation function using 3D LAS technique (Fenton and Vanmarcke 1990). The correlation coefficient between $k$ measured at a point $x_{1}$ and a second point $x_{2}$ is specified by a correlation function, $\rho(\tau)$, where $\tau=\left|x_{1}-x_{2}\right|$ is the absolute distance between the two points. An isotropic (i.e. the spatial correlation lengths in the horizontal and vertical directions are taken to be equal) exponentially decaying (Markovian) spatial correlation function is used in the current study, as follows (Fenton and Griffiths 2008):

$$
\rho(\tau)=\exp \left(-\frac{2|\tau|}{\theta_{k}}\right)
$$

It should be noted that, in natural soil deposits, the correlation structures in any spatial direction are often different (i.e. anisotropic) due to the complex process of weathering, transportation and soil layering. However, for the purpose of a generic non-site specific study, it is reasonable to assume that the spatial correlation function in Equation (1) to statistically isotropic for both $k$ and $m_{v}$. This means that the SOF in the horizontal direction $(x)$, the direction normal to the plane of paper $(y)$ and the vertical direction $(z)$ are the same (i.e. $\theta_{x}=\theta_{y}=\theta_{z}=\theta$ ). This assumption does not alter the general trend and observation presented in this study, hence, will not affect the basic understanding that might be acquired by considering anisotropic correlation structure. It is worthy to note that the spatial correlation length is estimated with respect to the underlying normally distributed random field.

Since $k$ is assumed to be characterized statistically by a lognormal distribution, the correlated standard normal random field, $G(x)$, generated using the LAS method is then transformed into a lognormal distribution by the following transformation function (Fenton and Griffiths 2008): 


$$
k_{i}=\exp \left\{\mu_{\ln k}+\sigma_{\ln k} G(i)\right\}
$$

where: $G(i)$ and $k_{i}$ are, respectively, the local (arithmetic) average of a standard Gaussian random field $G(x)$ over the domain of the $i$ 'th element and the soil property value assigned to that element; $\mu_{\ln k}$ and $\sigma_{\ln k}$ are the mean and standard deviation of the underlying normal distribution; $\mu_{\ln k}$ and $\sigma_{\ln k}$ are obtained from the specified permeability $\mu_{k}$ and $\sigma_{k}$ using the following lognormal distribution transformation functions (Fenton and Griffiths 2008):

$$
\begin{gathered}
\mu_{\ln k}=\ln \mu_{k}-\frac{1}{2} \sigma_{\ln k}^{2} \\
\sigma_{\ln k}=\sqrt{\ln \left(1+\frac{\sigma_{k}^{2}}{\mu_{k}^{2}}\right)}=\sqrt{\ln \left(1+v_{k}^{2}\right)}
\end{gathered}
$$

where: $v_{k}=\sigma_{k} / \mu_{k}$ is the permeability coefficient of variation (COV). It should be noted that the random fields of both $k$ and $m_{v}$ are generated using the 3D free access LAS computer code available online at http://www.engmath.dal.ca/rfem/.

\section{Finite-element modeling incorporating soil spatial variability}

With the complete subsurface profile having been simulated in the previous step, the spatial variability of $k$ and $m_{v}$ is now known and can be employed as input in a finite element (FE) consolidation modeling of soil improvement by PVDs. In this study, all numerical analyses are carried out using a modified version of the finite element computational scheme "Program 8.6" from the book by Smith and Griffiths (2004) in which soil consolidation is treated as a 3D uncoupled (i.e. no displacement degrees of freedom only pore pressure degrees of freedom) problem. Originally "Program 8.6" was for general two (plane) or three dimensional analyses of the uncoupled consolidation 
equation using implicit time integration with the "theta" method. The authors modified the source code of "Program 8.6" to allow for input of the volume compressibility and repetitive Monte-Carlo analyses. Since a single-drain analysis is often enough to investigate the soil consolidation behavior, the effect of soil spatial variability is examined using a unit cell of soil around a single drain. The consolidation problem considered in this study implies a unit cell (axisymmetric) of actual 3D geometry of PVD system (see Figure 1a): $L=1.0 \mathrm{~m}, r_{e}=0.8463 \mathrm{~m}, r_{s}=0.2821 \mathrm{~m}$ and $r_{w}=0.0637$ $\mathrm{m}$, where $L$ is the maximum vertical drainage distance; $r_{e}$ is the radius of equivalent soil cylinder with impermeable perimeter or the radius of zone of influence; $r_{s}$ is the radius of the smear zone; and $r_{w}$ is the equivalent radius of the drain. However for the finiteelement analyses, the circular influence area of the cylindrical unit cell is transformed into an equivalent square influence area (see Figure 1b) of side length $S$, such that $S=$ $\sqrt{ } \pi r_{e}{ }^{2}$ (i.e. $S=1.5 \mathrm{~m}$ ). The selection of square influence area instead of the equivalent circular influence area is to avoid the unfavorable mesh shape as the LAS method requires square (or rectangular) elements to accurately compute locally averaged values of $k$ and $m_{v}$ for each element across the grid. For the same reason, square shaped smear zone of side length $S_{s}=\sqrt{ } \pi r_{s}{ }^{2}$ and PVD of side length $S_{w}=\pi r_{w} / 2$ are employed. In order to check the validity of the transformation process of the actual cylindrical geometry to the "equivalent" square geometry for the FE analysis, a comparison is performed under the deterministic condition for the FE solution using a square mesh of an element size of $0.1 \mathrm{~m}$ and Hansbo's (1981) unit cell solution, and the results are shown in Figure 2. It can be seen that the two solutions are in good agreement despite the slight discrepancy at the earlier stage of consolidation, which may be attributed to the fact that the FE method is essentially a free strain analysis while Hansbo's (1981) solution is based on an equal strain assumption. It should be noted that, for simplicity, the well resistance 
factor which may affect the rate of consolidation is not considered in the FE analysis. This is due to the fact that the discharge capacities of most PVDs available in the market are relatively high, and hence the well resistance effect can be ignored in most practical cases (Abuel-Naga et al. 2012; Chu 2004). In soil stabilization by PVDs, soil consolidation takes place by combined vertical and horizontal (radial) drainage of water. However, for the case of PVDs, the overall consolidation is governed by the radial (horizontal) flow of water rather than the vertical flow as the drainage length in the horizontal direction is much less than that of the vertical direction and thus $k_{h}$ is often much higher than that of $k_{v}$ (Hansbo 1981). Under this reasoning, only the component of the overall consolidation resulted from the horizontal drainage is considered to be random in the current study. To simulate such condition, the permeability in the vertical $(z)$ direction, $k_{z}$ is set as to zero in the FE analysis. Since the permeability variance of even one of the directions is rarely known with any accuracy, the two components of the horizontal permeability (i.e. $k_{x}$ and $k_{y}$ ) are assumed as isotropic (i.e. $k_{x}=k_{y}$ ). In order to take the smear effect into consideration, two independent random fields of both $k$ and $m_{v}$ are generated separately (one for the smear zone and another for the undisturbed zone) employing the specified statistical parameters $(\mu, \sigma$ and $\theta)$ of each zone. Both random fields are then mapped onto the corresponding grid in the finite element mesh.

Generally speaking, discrepancy is inevitable in any discretization scheme of finite element (FE) modelling; however, it can be reduced by providing small elements in FE mesh. Although the accuracy of FE solutions increases with the increase of the number of elements in the mesh, a trade-off between accuracy and run-time efficiency is necessary due to limited computational resources. Previous literature includes some recommendations regarding the optimum ratio of the SOF to the size of finite elements. 
For example, Ching and Phoon (2013) stated that this ratio should be $\geq 20$, whereas Harada and Shinozuka (1986) pointed out that it should be $\geq 2$. In the current study, a sensitivity analysis on two different FE meshes with element sizes of $0.1 \mathrm{~m}$ and $0.05 \mathrm{~m}$ is conducted. For a certain SOF, two random fields for the two selected meshes are generated with the same seed. The degree of consolidation is computed from the subsequent FE analysis for both random fields and checked whether that they are nearly identical or not. Several different random seeds and SOFs are tested, for permeability coefficient of variation $v_{k_{h}}=200 \%$ and compressibility coefficient of variation $v_{m_{v}}=$ $30 \%$ and the results are presented in Figure 3. It can be seen that for certain seed and SOF, $U(t)$ obtained from the two meshes of element sizes of $0.1 \mathrm{~m}$ and $0.05 \mathrm{~m}$ are almost identical with the only exception when SOF is as low as $0.125 \mathrm{~m}$, which complies with the recommendation given by Harada and Shinozuka (1986). Based on this observation and in order to comply with the minimum correlation length used, a mesh with an element size of $0.1 \mathrm{~m} \times 0.1 \mathrm{~m} \times 0.1 \mathrm{~m}$ (see Figure $1 \mathrm{~b}$ ) is adopted in the current study. It should be noted that the 3D mesh used consists of 2250 eight node first order hexahedral elements and remained fixed for all selected SOF. Therefore, the minimum and maximum ratios of SOF to FE size are thus equal to 2.5 and 100, respectively (the minimum and maximum SOFs are chosen to be $0.25 \mathrm{~m}$ and $10.0 \mathrm{~m}$, respectively). As the discrepancy in the FE solutions decreases when the ratio of SOF to FE size increases, a systematic bias might exist in the results presented in this study particularly for very small SOF but diminishes as the SOF increases.

To simulate reduced permeability condition in the smear zone during the FE analysis, the mean values of $k$ in the undisturbed and smear zones are taken to be equal to $\mu_{k_{u}}=0.03 \mathrm{~m} /$ year and $\mu_{k_{s}}=0.015 \mathrm{~m} /$ year, respectively, which means that $\mu_{k_{u}} / \mu_{k_{s}}=$ 2.0. This is because, on the basis of laboratory experiments carried out on Bangkok 
clay, Bergado et al. (1991) reported that $k_{h} / k_{s}$ is approximately 1.5-2.0. on the other hand, Indraratna and Redana (1998) also reported that $k_{h} / k_{s}$ is in the range of $2-3$. Moreover, Terzaghi et al. (1996) stated that a ratio of $k_{h} / k_{s}$ equal to 2.0 is usually assumed when there are no experimental data available. Walker (2006) indicates that the value of the smear zone compressibility could increase by about $20 \%$ from that of the undisturbed zone. Therefore, to consider increased compressibility condition in the smear zone, the mean value of $m_{v}$ in the undisturbed and smear zones are taken to be equal to $\mu_{m_{v_{u}}}=8.0 \times 10^{-4} \mathrm{~m}^{2} / \mathrm{kN}$ and $\mu_{m_{v_{s}}}=9.6 \times 10^{-4} \mathrm{~m}^{2} / \mathrm{kN}$, respectively, which means that $\mu_{m_{v_{s}}} / \mu_{m_{v_{s}}}=1.2$. The effect of spatially variable of $k$ and $m_{v}$ on the stochastic behavior of soil consolidation by PVDs is investigated over a range of different combinations of standard deviation, $\sigma$, and scale of fluctuation, $\theta$. As stated earlier, both $k$ and $m_{v}$ are assumed to be lognormally distributed. It should be noted that $\sigma$ is presented herein in a normalized form as $v$ (i.e. coefficient of variation). The following values of $v$ and $\theta$ are considered for the parametric study presented in this paper:

- $v_{k}($ for both smear and undisturbed zones $)=50,100,200(\%)$

- $v_{m_{v}}($ for both smear and undisturbed zones $)=10,20,30(\%)$

- $\theta$ (for both $k$ and $m_{v}$, and smear and undisturbed zones) $=0.25,0.5,1,5,10(\mathrm{~m})$

It can be noticed that, the selected range of COV of $m_{v}$ is much less than that of the range selected for COV of $k$. This is due to the fact that $k$ is considered to be the most significant spatially random soil property affecting soil consolidation, with COV as high as $300 \%$, while $m_{v}$ usually possess COV of up to $30 \%$ as reported in the literature (e.g. Beacher and Christian 2003; Kulhawy et al. 1991; Lee et al. 1983). However, the range of $\theta$ is assumed to be the same for both $k$ and $m_{v}$. This assumption is reasonable 
because, if one thinks that the spatial correlation structure of a soil is caused by changes in the constitutive nature of the soil over the ground, then both $k$ and $m_{v}$ would have similar correlation lengths. Since little is currently known about the typical COVs and SOFs of soils in the smear zone, the same range of $v$ and $\theta$ are selected for both smear and undisturbed zones. It should be noted that $k_{h}$ and $m_{v}$ are assumed to be uncorrelated in this study, which is due to the lack of data available in the literature to identify the nature and level of correlation between $k$ and $m_{v}$. For the problem of one dimensional consolidation, Freeze (1977) reported that non-zero cross-correlation between $k$ and $m_{v}$ has a minor impact on the stochastic results of soil consolidation. In addition, the introduction of cross-correlation decreases the variability between $k$ and $m_{v}$. In other words, the assumption of independence between $k$ and $m_{v}$ increases the overall variability held in the model which leads to a slightly conservative results. Under this reasoning, $k$ and $m_{v}$ are assumed to be independent in the current study rather than correlated, which is deemed to be reasonable instead of assuming any erroneous correlation.

In order to identify the statistical parameters in the smear and undisturbed zones, $v$ and $\theta$ of $k$ and $m_{v}$ are denoted with appropriate subscripts " $s$ " and " $u$ " depending on whether they are specified for smear zone or undisturbed zone, where $s$ refers to the smear zone while $u$ refers to undisturbed zone. An initial pore water pressure of $100 \mathrm{kPa}$ dissipates in a single drain is considered in all FE analyses. A single generation of a random field and the subsequent finite-element analysis of that field are termed "realization". For an individual realization, the degree of consolidation, $U(t)$, at any certain consolidation time, $t$, is calculated with the help of the following expression:

$$
U(t)=1-\frac{\bar{u}(t)}{u_{0}}
$$


where: $u_{0}=$ initial pore pressure; and $\bar{u}(t)=$ average pore pressures at any time of the consolidation process. It has to be emphasized that $\bar{u}(t)$ of the consolidation process is calculated by numerically integrating the pore pressure across the volume of each element at a particular time, summing the contribution of each element and dividing by the total mesh volume (element volume are also calculated by numerical integration).

\section{Repetition of process based on the Monte Carlo technique}

Following the procedures of the Monte Carlo technique, the process of generating random fields of $k$ and $m_{v}$ and performing the finite element analysis is repeated numerous times until an acceptable accuracy of the estimated statistics of $U(t)$ is achieved. It was found that 2000 realizations are sufficient to give reasonably stable output statistics for the first two moments (i.e. $\mu_{U}$ and $\sigma_{U}$ ) of the degree of consolidation. The obtained outputs from the suite of 2000 realizations of the Monte Carlo simulation are collated and statistically analyzed to produce estimates of the mean and standard deviation of the degree of consolidation. In this study, at any given time $t$, the mean of the degree of consolidation based on the excess pore water pressure, $\mu_{U}$, is estimated by utilizing the geometric average (considered as the representative mean) of $\bar{u}(t)$, as follows:

$$
\mu_{U}=1-\exp \left[\frac{1}{n_{\text {sim }}} \sum_{i=1}^{n_{\text {sim }}} \ln \left(\frac{\bar{u}(t)}{u_{0}}\right)_{i}\right]
$$

The standard deviation of the average degree of consolidation at any time $t$ defined by the pore water pressure, $\sigma_{U}$, is estimated as follows:

$$
\sigma_{U}=\sqrt{\frac{1}{n_{\text {sim }}-1} \sum_{i=1}^{n_{\text {sim }}}\left[(U(t))_{i}-\mu_{U}\right]^{2}}
$$


where: $n_{\text {sim }}$ is the number of Monte Carlo simulations; $\left(\bar{u}(t) / u_{0}\right)_{i}$ and $(U(t))_{i}$ are, respectively, the ratio of the average excess pore pressure to the initial excess pore water pressure and the degree of consolidation at any time $t$ for the $i$ th simulation (see Equation (5)). The use of the geometric average for $\bar{u}(t)$ in computing $\mu_{U}$ is due to the fact that the flow of water in $2 \mathrm{D}$ or $3 \mathrm{D}$ spaces compared to the 1D space has more freedom to avoid low permeability zones by detouring around them and therefore the geometric average may be a better estimator (e.g. Dagan 1989) for computing the representative mean of the average excess pore water pressures. For the same reason, Huang et al. (2010) also used the geometric average in determining the equivalent coefficient of consolidation for a 2D system.

\section{Probabilistic interpretation}

The estimation of the probability that a deterministic degree of consolidation overestimates the true consolidation value is one of the main objectives of the stochastic consolidation analyses. Such probability can be represented either by the probability of achieving a target degree of consolidation, $U_{s}$, (i.e. $\left.P\left[U\left(t_{s}\right) \geq U_{s}\left(t_{s}\right)\right]\right)$ at any specified consolidation time, $t_{s}$, or the probability of required time $t$ to achieve $U_{s}$ that is less than or equal to $t_{s}$ (i.e. $\left.P\left[t\left(U_{s}\right) \leq t_{s}\left(U_{s}\right)\right]\right)$. In this study, the later process is employed, i.e. $P\left[t\left(U_{s}\right) \leq t_{s}\left(U_{s}\right)\right]$ is estimated. This is because determining probability from a set of data requires establishment of a reasonable probability distribution for the data set. However, the obtained fit using the raw data of $U\left(t_{s}\right)$ was typically poor while the distribution of $t\left(U_{s}\right)$ obtained from the suite of the 2000 realizations is reasonably fitted with lognormal distribution and gives sufficiently reasonable approximation to the $P\left[t\left(U_{s}\right) \leq t_{s}\left(U_{s}\right)\right]$. The legitimacy of the lognormal distribution hypothesis for $t\left(U_{s}\right)$ is examined by the wellknown Chi-square test through the frequency density plot of $t\left(U_{s}\right)$ data obtained from 
the 2000 realizations and a fitted lognormal distribution is superimposed. This process is performed for many combinations of $v$ and $\theta$ at several different $U_{s}$. For each of the cases considered, the goodness-of-fit $p$-value is found to be high enough to support the rationality of the lognormal distribution hypothesis of simulated $t\left(U_{s}\right)$ data. Figure 4 illustrates a typical example of the histogram of $t\left(U_{s}\right)$ for the case of $v_{k_{u}}=50 \%, v_{k_{s}}=$ $200 \%, v_{m_{v_{u}}}=10 \%, v_{m_{v_{s}}}=30 \%, \theta_{k_{u}}=\theta_{k_{s}}=\theta_{m_{v_{u}}}=\theta_{m_{v_{u}}}=0.5 \mathrm{~m}$ at $U_{s}=90 \%$, along with their fitted lognormal distributions. The goodness-of-fit test at 5\% significance (equivalent to $95 \%$ confidence) level yielded $p$-value of 0.4 , indicating that there is very little evidence in the simulated $t\left(U_{s}\right)$ sample against the null hypothesis.

By accepting the lognormal distribution for $t$ at any given $U_{s}$, the statistical moments $\mu_{t\left(U_{s}\right)}$ and $\sigma_{t\left(U_{s}\right)}$ that are representing the mean and standard deviation of the lognormally distributed $t$ that achieves $U_{s}$ are calculated from the suite of 2000 realizations using the following transformation functions:

$$
\begin{gathered}
\mu_{t\left(U_{s}\right)}=\frac{1}{n_{\text {sim }}} \sum_{i=1}^{n_{\text {sim }}} t_{i}\left(U_{s}\right) \\
\sigma_{t\left(U_{s}\right)}=\sqrt{\frac{1}{n_{\text {sim }}-1} \sum_{i=1}^{n_{\text {sim }}}\left[t_{i}\left(U_{s}\right)-\mu_{t\left(U_{s}\right)}\right]^{2}}
\end{gathered}
$$

where: $t_{i}\left(U_{s}\right)$ is the $t$ from the $i$ 'th realization $\left(i=1,2,3, \ldots, n_{\text {sim }}\right)$ at given $U_{s}$ and $n_{\text {sim }}=$ total number of realizations $=2000$. As $90 \%$ consolidation is usually acceptable for the purpose of design of any soil improvement project (Bo et al. 2003), in this study, it is assumed that the target degree of consolidation is $90 \%$ and for convenience, it is simply denoted as $U_{90}$. The probability that $t$ is less than or equal to $t_{s}$ that achieves $U_{90}$ can then be obtained from the following lognormal probability distribution transformation: 


$$
P\left[t\left(U_{90}\right) \leq t_{s}\left(U_{90}\right)\right]=\Phi\left(\frac{\ln t_{s}-\mu_{\ln t\left(U_{90}\right)}}{\sigma_{\ln t\left(U_{90}\right)}}\right)
$$

where: $P[$.$] is the probability of its argument; \Phi($.$) is the standard normal cumulative$ distribution function; $\mu_{\ln t\left(U_{S}\right)}$ and $\sigma_{\ln t\left(U_{S}\right)}$ are, respectively, the mean and standard deviation of the underlying normally distributed $\ln t\left(U_{s}\right)$ and can be estimated from $\mu_{t\left(U_{S}\right)}$ and $\sigma_{t\left(U_{S}\right)}$ with reference to Equations (3) and (4), as follows:

$$
\begin{gathered}
\mu_{\ln t\left(U_{s}\right)}=\ln \mu_{t\left(U_{s}\right)}-\frac{1}{2} \sigma_{\ln t\left(U_{s}\right)}^{2} \\
\sigma_{\ln t\left(U_{s}\right)}=\sqrt{\ln \left(1+\frac{\sigma_{t\left(U_{s}\right)}^{2}}{\mu_{t\left(U_{s}\right)}^{2}}\right)}
\end{gathered}
$$

Following the procedure set out above, probabilities of required time $t$ to achieve $U_{s}$ that is less than or equal to $t_{s}$ can be estimated for any combination of $v$ and $\theta$, and the stochastic behaviour of soil consolidation by PVDs can be investigated.

\section{Results and discussion}

In order to investigate the relative significance of the spatially variability of the smear zone over the undisturbed zone and to identify the random variable that has the major contribution to the uncertainty of the degree of consolidation, a series of 3D consolidation analyses are performed. The sensitivity of the statistics of the degree of consolidation and the probability of required time $t$ to achieve $U_{s}$ that is less than or equal to $t_{s}$ to the statistically defined input data (i.e. $v$ and $\theta$ ) in relation to both $k$ and $m_{v}$ is examined. For each selected set of $v$ and $\theta, 2000$ Monte Carlo simulations are performed. The obtained consolidation responses are then statistically analyzed to estimate $\mu_{U}, \sigma_{U}$ and $P\left[t\left(U_{90}\right) \leq t_{s}\left(U_{90}\right)\right]$ using the excess pore water pressure. Since the 
general trends of $\mu_{U}, \sigma_{U}$ and $P\left[t\left(U_{90}\right) \leq t_{s}\left(U_{90}\right)\right]$ remain unaltered over the specified range of $v$ and $\theta$, only the results of a few of the tests conducted are presented in Figures 5-11, which are believed to be sufficient to demonstrate the main features of the influence of spatial variability of $k$ and $m_{v}$ on soil consolidation by PVDs. In Figures 5-11, $\mu_{U}, \sigma_{U}$ and $P\left[t\left(U_{90}\right) \leq t_{s}\left(U_{90}\right)\right]$ are expressed as a function of time $t$. Prior to placing the stochastic analyses into context, an initial deterministic solution has been performed assuming a homogeneous soil. It should be noted that the deterministic solution of this case yields $U_{90}$ at $t=0.73$ year (i.e. $t_{\mathrm{D} 90}=0.73$ year). The results obtained from this study are described below.

\section{Effect of variation of $v$ and $\theta$ on the mean of $U$}

The effects of increasing $v_{u}$ and $v_{s}$ on $\mu_{U}$ at fixed value of $\theta_{u}=\theta_{s}=0.5 \mathrm{~m}$ is examined in Figure 5, which also includes the deterministic solution of no soil variability. It can be seen from Figure 5a that at any consolidation time, there is a slight reduction in $\mu_{U}$ for spatially varied soils compared to the deterministic case. The nearly identical curves for all cases of $v_{u}\left(v_{k_{s}}\right.$ and $v_{m_{v_{S}}}$ are fixed at $50 \%$ and $10 \%$ respectively) plotted in the figure indicate that the effect of increasing $v_{u}$ on $\mu_{U}$ remains marginal. The effect of $v_{s}$ on $\mu_{U}$ at fixed values of $v_{k_{u}}=50 \%$ and $v_{m_{v}}=10 \%$ is illustrated in Figure $5 \mathrm{~b}$, which shows that any change in $v_{s}$ has a significant impact on the estimated values of $\mu_{U}$. At any certain consolidation time, $\mu_{U}$ decreases with the increase of $v_{s}$, and the decreasing rate of $\mu_{U}$ consistently increases with the increase of $v_{s}$. The comparison between Figures $5 \mathrm{a}$ and $5 \mathrm{~b}$ reveals that the effect of $v_{s}$ on $\mu_{U}$ is dominating.

Figure 6 highlights the effects of increasing $\theta_{u}$ and $\theta_{s}$ on $\mu_{U}$ at fixed values of $v_{k_{u}}=v_{k_{s}}=50 \%$ and $v_{m_{v_{u}}}=v_{m_{v_{s}}}=10 \%$. Virtually, the identical curves of $\mu_{U}$ in Figure 6a for all $\theta_{u}$ at a fixed value of $\theta_{k_{s}}=\theta_{m_{v_{s}}}=0.25 \mathrm{~m}$, indicate that $\mu_{U}$ is more or less 
independent of $\theta_{u}$. Similar to $\theta_{u}$, the influence of $\theta_{s}\left(\theta_{k_{u}}\right.$ and $\theta_{m_{v_{u}}}$ are fixed at $\left.0.25 \mathrm{~m}\right)$ on $\mu_{U}$ is also marginal as can be seen in Figure $6 \mathrm{~b}$. In general, it can be observed that even though the results for various $\theta$ are drawn in Figure 6, they are embodied into a single curve, implying that the obtained results at different $\theta$ are very close and cannot be distinguished. The virtually identical curves for all $\theta$ at each plot demonstrate that $\mu_{U}$ is largely independent of $\theta$. This is expected as in principle $\theta$ does not affect the local average mean of the process.

\section{Effect of variation of $v$ and $\theta$ on the standard deviation of $U$}

The influence of $v_{u}$ and $v_{s}$ on $\sigma_{U}$ at a fixed value of $\theta_{u}=\theta_{s}=0.5 \mathrm{~m}$ is depicted in Figure 7. For a fixed value of $v_{s}\left(v_{k_{s}}\right.$ and $v_{m_{v_{S}}}$ are, respectively, $50 \%$ and $10 \%$ in this case), increasing $v_{u}$ has a marginal effect on $\sigma_{U}$, as shown in Figure 7a. Figure $7 \mathrm{~b}$ shows the effect of $v_{s}$ on $\sigma_{U}$ at fixed values of $v_{k_{u}}=50 \%$ and $v_{m_{v_{u}}}=10 \%$, and from which it can be seen that at any certain consolidation time, $\sigma_{U}$ increases significantly with the increase of $v_{s}$, implying the dominant effect of $v_{s}$ on the estimated values of $\sigma_{U}$.

Figure 8 illustrates the effect of varying $\theta_{u}$ and $\theta_{s}$ on $\sigma_{U}$ at fixed values of $v_{k_{u}}=$ $v_{k_{s}}=50 \%$ and $v_{m_{v_{u}}}=v_{m_{v_{s}}}=10 \%$. In Figure $8 \mathrm{a}$, it can be seen that similar to the effect of $\theta_{u}$ on $\mu_{U}, \sigma_{U}$ remains almost identical for varying $\theta_{u}$ with a fixed value of $\theta_{k_{s}}=\theta_{m_{v_{s}}}=$ $0.25 \mathrm{~m}$. On the other hand, the estimated $\sigma_{U}$ for different values of $\theta_{s}$ is plotted in Figure $8 \mathrm{~b}$ at a fixed value of $\theta_{k_{u}}=\theta_{m_{v_{u}}}=0.25 \mathrm{~m}$, which illustrates that unlike $\theta_{u}, \theta_{s}$ has a considerable impact on the estimated values of $\sigma_{U}$. It can also be seen that for the consolidation problem under consideration, the increasing rate in $\sigma_{U}$ with the increase of $\theta$ becomes insensitive when $\theta \geq 5.0 \mathrm{~m}$. 


\section{Effect of variation of $v$ and $\theta$ on the probability of required time t to achieve $U_{90}$ that is less than or equal to $t_{s}$}

The influence of the smear zone parameters over the undisturbed zone parameters in relation to the probability of required time $t$ to achieve $U_{90}$ that is less than or equal to $t_{s}$ are investigated in Figures 9 and 10. The deterministic time of achieving 90\% consolidation, $t_{\mathrm{D} 90}$, is also shown in the figures by vertical solid lines that give $P\left[t\left(U_{90}\right)\right.$ $\left.\leq t_{s}\left(U_{90}\right)\right]$ at that time, for any combination of $v$ and $\theta$.

The effects of $v_{u}$ and $v_{s}$ on $P\left[t\left(U_{90}\right) \leq t_{s}\left(U_{90}\right)\right]$ at a fixed value of $\theta_{u}=\theta_{s}=0.5 \mathrm{~m}$ is demonstrated in Figure 9. It can be seen from Figure 9a that, in general, the effect of increasing $v_{u}\left(v_{k_{s}}\right.$ and $v_{m_{v_{s}}}$ are fixed at $50 \%$ and $10 \%$, respectively) on $P\left[t\left(U_{90}\right) \leq\right.$ $\left.t_{s}\left(U_{90}\right)\right]$ remains marginal. The effect of $v_{s}$ at fixed values of $v_{k_{u}}=50 \%$ and $v_{m_{v_{u}}}=10 \%$ is shown in Figure 9b, which shows that varying the values of $v_{s}$ has a considerable impact on the estimated values of $P\left[t\left(U_{90}\right) \leq t_{s}\left(U_{90}\right)\right]$. At any certain consolidation time, $P\left[t\left(U_{90}\right) \leq t_{s}\left(U_{90}\right)\right]$ decreases significantly with the increase of $v_{s}$. The overall observation that can be derived from comparing the results in Figure 9 is that the effect of $v_{s}$ on $P\left[t\left(U_{90}\right) \leq t_{s}\left(U_{90}\right)\right]$ is dominant.

Figure 10 investigates the effects of $\theta$ on $P\left[t\left(U_{90}\right) \leq t_{s}\left(U_{90}\right)\right]$ at fixed values of $v_{k_{u}}=v_{k_{s}}=50 \%$ and $v_{m_{v_{u}}}=v_{m_{v_{s}}}=10 \%$. In Figure 10a, the influence of increasing $\theta_{u}$ on $P\left[t\left(U_{90}\right) \leq t_{s}\left(U_{90}\right)\right]$ is shown at $\theta_{k_{s}}=\theta_{m_{v_{s}}}=0.25 \mathrm{~m}$, and the results yield almost identical curves indicating that varying the values of $\theta_{u}$ has little or no impact on the probabilistic behavior of degree of consolidation. On the other hand, the estimated $P\left[t\left(U_{90}\right) \leq t_{s}\left(U_{90}\right)\right]$ for different values of $\theta_{s}$ is plotted in Figure $10 \mathrm{~b}$ at a fixed value of $\theta_{k_{u}}=\theta_{m_{v_{u}}}=0.25 \mathrm{~m}$. It can be seen that unlike $\theta_{u}, \theta_{s}$ has a considerable impact on the estimated values of $P\left[t\left(U_{90}\right) \leq t_{s}\left(U_{90}\right)\right]$; however, at any certain consolidation time $P\left[t\left(U_{90}\right) \leq t_{s}\left(U_{90}\right)\right]$ becomes insensitive to $\theta_{s}$ when $\theta_{s} \geq 5.0 \mathrm{~m}$. The comparison between 
Figures $10 \mathrm{a}$ and $10 \mathrm{~b}$ reveals that, the effect of $\theta_{s}$ on $P\left[t\left(U_{90}\right) \leq t_{s}\left(U_{90}\right)\right]$ is more significant than $\theta_{u}$. It is interesting to know that the deterministic solution yields $P\left[t\left(U_{90}\right) \leq t_{s}\left(U_{90}\right)\right]<50 \%$ for all combinations of values of $v_{u}, v_{s}, \theta_{u}$, and $\theta_{s}$, as can be seen in Figures 9 and 10.

\section{Effect of the degree of variability of $m_{v}$ on $\mu_{U}, \sigma_{U}$ and $P\left[t\left(U_{90}\right) \leq t_{s}\left(U_{90}\right)\right]$ of $U$}

In order to determine the relative contribution of spatially variable $k$ and $m_{v}$ to the uncertainty of the degree of consolidation at any certain consolidation time, a sensitivity analysis consists of a total of five stochastic simulation tests is performed. The COV of $k$ is fixed at $100 \%$ in all five tests, while the COV of $m_{v}$ is varied as $25 \%, 33 \%, 50 \%$, $100 \%$ in addition to one simulation test with no variability in $m_{v}$ (i.e. homogeneous with $\mu_{m_{v_{u}}}$ and $\left.\mu_{m_{v_{s}}}\right)$. The specified COV for $k$ and $m_{v}$ is kept the same for both the undisturbed and smear zones. A fixed value of $\theta_{u}=\theta_{s}=0.5 \mathrm{~m}$ is considered for $k$ and $m_{v}$. The results obtained from this sensitivity analysis are shown in Figure 11. It can be seen that $\mu_{U}$ (Figure 11a), $\sigma_{U}$ (Figure 11b) and $P\left[t\left(U_{90}\right) \leq t_{s}\left(U_{90}\right)\right]$ (Figure 11c) are almost identical for each conducted test implying that the variability of $m_{v}$ has a little or no effect on the estimated $\mu_{U}, \sigma_{U}$ and $P\left[t\left(U_{90}\right) \leq t_{s}\left(U_{90}\right)\right]$ based on excess pore pressure. However, when $v_{m_{v}} \approx v_{k}$, a noticeable effect is found. For this case, $\mu_{U}, \sigma_{U}$ and $P\left[t\left(U_{90}\right) \leq t_{s}\left(U_{90}\right)\right]$ are slightly higher than those obtained from the case with homogeneous $m_{v}$. Therefore, the variability of $m_{v}$ has a negligible impact on the degree of consolidation estimated based on the excess pore pressure. Consequently, for the uncoupled stochastic analysis of soil consolidation by PVDs, $m_{v}$ can be considered to be deterministic (i.e. spatially constant). It should be emphasised that the possible reduction in the number of spatially variable parameters allows the superfluous 
complexity of the stochastic problem to be reduced in conjunction with the computation time. It may also reduce the cost of the site investigation program required for characterizing the soil spatial variability.

\section{Conclusions}

This paper used the random field theory and finite element modeling to investigate the relative significance of soil spatially variability in the smear and undisturbed zones in soil improvement by prefabricated vertical drains. A sensitivity analysis was also performed to identify the most significant random variable affecting stochastic response of soil consolidation by PVDs. The coefficient of permeability, $k$, and coefficient of volume compressibility, $m_{v}$, were treated as independent random variables and uncoupled 3D finite element analysis was applied. The effect of coefficient of variation, $v$, and spatial correlation or scale of fluctuation, $\theta$, of the undisturbed zone on the estimated mean and standard deviation of the degree of consolidation was found to be marginal. On the other hand, the estimated statistics and probability associated with the degree of consolidation were found to be highly sensitive to $v$ and $\theta$ of the spatially variable soil properties at the smear zone. This result indicates that the probabilistic behavior of soil consolidation is governed by the spatial variation of the soil properties of the smear zone. Since the spatial variability of the smear zone will possibly be different from that of the undisturbed zone, this observation has important implications in the sense that, modeling soil consolidation with the same $v$ and $\theta$ for both zones (i.e. undisturbed and smear) that are equal to the $v$ and $\theta$ of the smear zone does not significantly affect the final results. It is also found that the variability of $m_{v}$ has a negligible contribution to the uncertainty of the degree of consolidation estimated via the uncoupled stochastic analysis, thus, can be considered to be deterministic (i.e. 
spatially constant). This is an important observation from the point of view that the reduction in the number of spatially variable parameters not only reduces the computation time but also minimizes the cost of the site investigation program required to establish the soil variability characterization. Overall, the results obtained from this research highlight valuable insights into the impact of soil spatial variability on soil improvement by PVDs and clearly demonstrate the potential of stochastic analyses in routine design practice.

\section{References}

Abuel-Naga, H. M., Pender, M. J., and Bergado, D. T., 2012. Design curves of prefabricated vertical drains including smear and transition zones effects. Geotextiles and Geomembranes, 32, 1-9.

Badaoui, M., Nour, A., Slimani, A., and Berrah, M. K., 2007. Consolidation statistics investigation via thin layer method analysis. Transport in Porous Media, 67(1), 69-91.

Bari, M. W., Shahin, M. A., and Nikraz, H. R., 2012. Effects of soil spatial variability on axisymmetric versus plane strain analyses of ground improvement by prefabricated vertical drains. International Journal of Geotechnical Engineering, 6(2), 139-147.

Bari, M. W., Shahin, M. A., and Nikraz, H. R., 2013. Probabilistic analysis of soil consolidation via prefabricated vertical drains. International Journal of Geomechanics, ASCE, 13(6), 877-881.

Beacher, G. B., and Christian, J. T., 2003. Reliability and Statistics in Geotechnical Engineering. John Wiley \& Sons, Chichester, England.

Bergado, D. T., Asakami, H., Alfaro, M. C., and Balasubramaniam, A. S., 1991. Smear effects of vertical drains on soft Bangkok clay. Journal of Geotechnical Engineering, ASCE, 117(10), 1509-1530.

Bo, M. W., Chu, J., Low, B. K., and Choa, V., 2003. Soil Improvement: Prefabricated Vertical Drain Techniques. Thomson Learning, Singapore.

Ching, J., and Phoon, K.-K., 2013. Effect of element sizes in random field finite element simulations of soil shear strength. Computers \& structures, 126(1), 120134.

Chu, J., 2004. Practical considerations for using vertical drains in soil improvement projects. Geotextiles and Geomembranes, 22(1), 101-117. 
Dagan, G., 1989. Flow and transport in porous media. Springer, New York.

Elkateb, T., Chalaturnyk, R., and Robertson, P. K., 2003. An overview of soil heterogeneity: quantification and implications on geotechnical field problems. Canadian Geotechnical Journal, 40(1), 1-15.

Fenton, G. A., and Griffiths, D. V., 2008. Risk assessment in geotechnical engineering. Wiley, New York.

Fenton, G. A., and Vanmarcke, E. H., 1990. Simulation of random fields via local average subdivision. Journal of Engineering Mechanics, 116(8), 1733-1749.

Freeze, R. A., 1977. Probabilistic one-dimensional consolidation. Journal of Geotechnical Engineering Division, 103(GT7), 725-742.

Hansbo, S., 1981. Consolidation of fine-grained soils by prefabricated drains. Proceedings of the 10th International Conference on Soil Mechanics and Foundation Engineering, Stockholm, Sweden, 677-682.

Harada, T., and Shinozuka, M., 1986. The scale of correlation for stochastic fields. Technical Report, Department of Civil Engineering and Engineering Mechanics, Columbia University, New York.

Hong, H. P., and Shang, J. Q., 1998. Probabilistic analysis of consolidation with prefabricated vertical drains for soil improvement. Canadian Geotechnical Journal, 35(4), 666-677.

Houmadi, Y., Ahmed, A., and Soubra, A.-H., 2012. Probabilistic analysis of a onedimensional consolidation problem. Georisk: Assessment and Management of Risk for Engineered Systems and Geohazards, 6(1), 36-49.

Huang, J., and Griffiths, D. V., 2010. One-dimensional consolidation theories for layered soil and coupled and uncoupled solutions by finite-element method. Géotechnique, 60(9), 709-713.

Huang, J., Griffiths, D. V., and Fenton, G. A., 2010. Probabilistic analysis of coupled soil consolidation. Journal of Geotechnical and Geoenvironmental Engineering, 136(3), 417-430.

Hwang, D., and Witczak, M. W., 1984. Multidimensional probabilistic consolidation. Journal of Geotechnical Engineering, 110(8), 1059-1077.

Indraratna, B., and Redana, I. W., 1998. Laboratory determination of smear zone due to vertical drain installation. Journal of Geotechnical and Geoenvironmental Engineering, ASCE, 124(2), 180-185.

Jaksa, M. B., Brooker, P. I., and Kaggwa, W. S., 1997. Inaccuracies associated with estimating random measurement errors. Journal of Geotechnical and Geoenvironmental Engineering, 123(5), 393-401.

Kulhawy, F. H., Roth, M. J. S., and Grigoriu, M. D., 1991. Some statistical evaluations of geotechnical properties. Proceedings of the 6th International Conference on 
Applied Statistical Problems in Civil Engineering (ICASP6), Mexico City, 705712.

Lee, I. K., White, W., and Ingles, O. G., 1983. Geotechnical engineering. Pitman, London.

Lee, P. K., Xie, K. H., and Cheung, Y. K., 1992. A study on one-dimensional consolidation of layered systems. International Journal of Numerical and Analytical Methods in Geomechanics, 16(11), 815-831.

Pyrah, I. C., 1996. One-dimensional consolidation of layered soils. Géotechnique, 46(3), 555-560.

Rowe, P. W., 1972. The relevance of soil fabric to site investigation practice. Géotechnique, 22(2), 195-300.

Shahin, M. A., and Bari, M. W., 2012. Modeling of ground improvement by prefabricated vertical drains in highly variable soils. International Conference on Ground Improvement and Ground Control (ICGI), University of Wollongong, Australia, 321-335.

Smith, I. M., and Griffiths, D. V., 2004. Programming the finite element method. John Wiley and Sons.

Terzaghi, K., Peck, R. B., and Mesri, G., 1996. Soil mechanics in engineering practice. Wiley Interscience, New York.

Vanmarcke, E. H., 1977. Probabilistic modelling of soil profiles. Journal of Geotechnical Engineering Division, 103(11), 1227-1246.

Walker, R. T., 2006. Analytical solutions for modeling soft soil consolidation by vertical drains. Thesis $(\mathrm{PhD})$. University of Wollongong, Wollongong, Australia.

Zhou, W., Hong, H. P., and Shang, J. Q., 1999. Probabilistic design method of prefabricated vertical drains for soil improvement. Journal of Geotechnical and Geoenvironmental Engineering, 125(8), 659-664. 

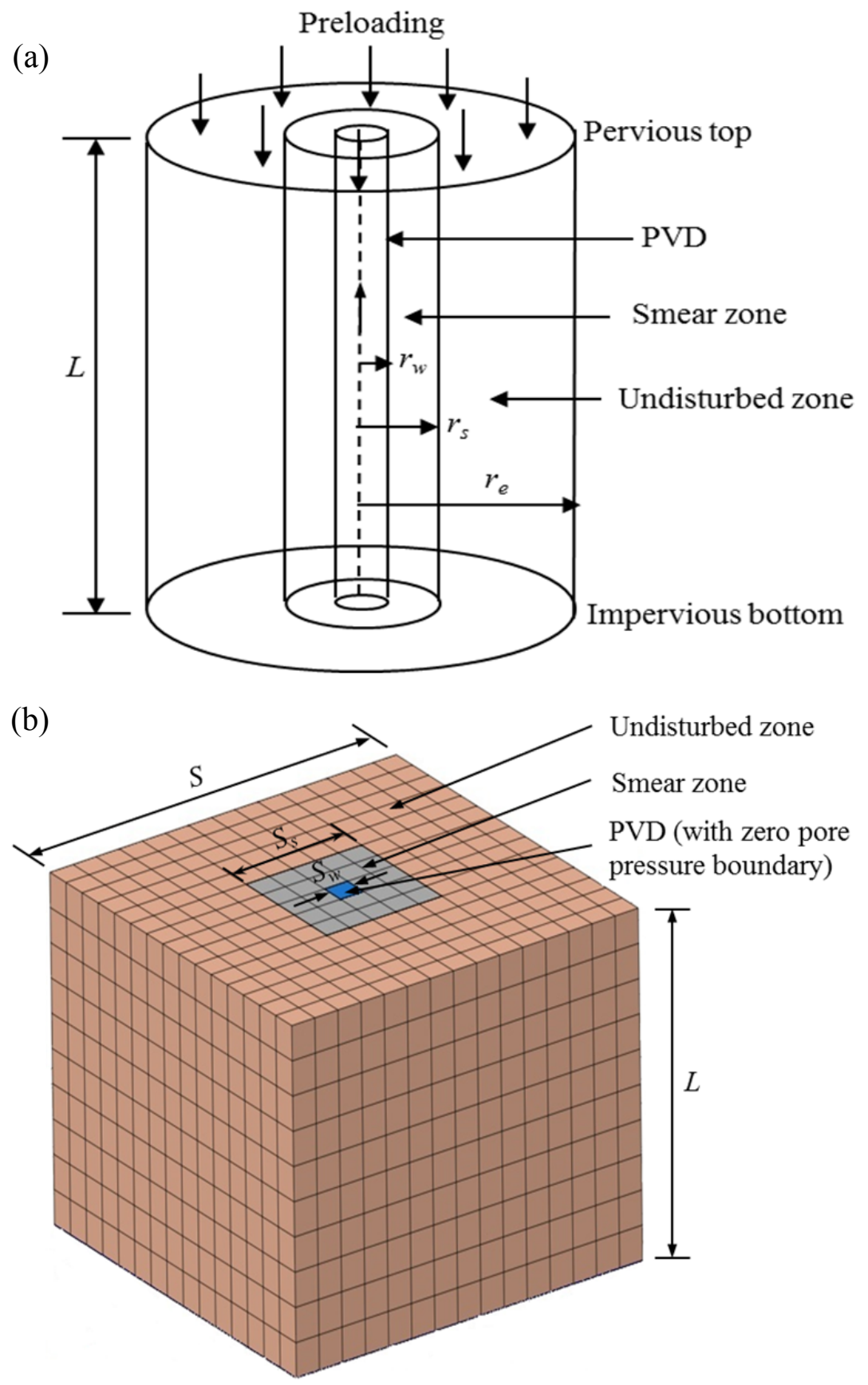

Figure 1. Schematic diagram of soil consolidation with prefabricated vertical drain: (a) cylindrical unit cell; (b) equivalent square geometry with FE mesh discretization. 


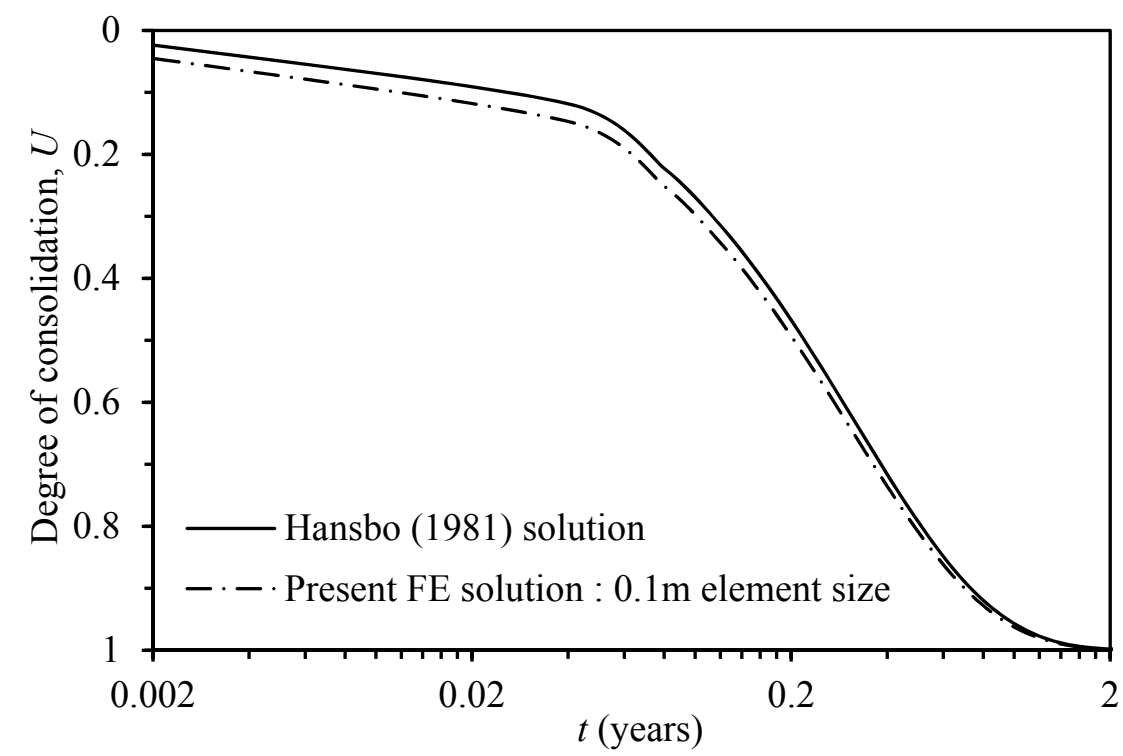

Figure 2. Comparison between finite element (square mesh) and Hansbo's solution.

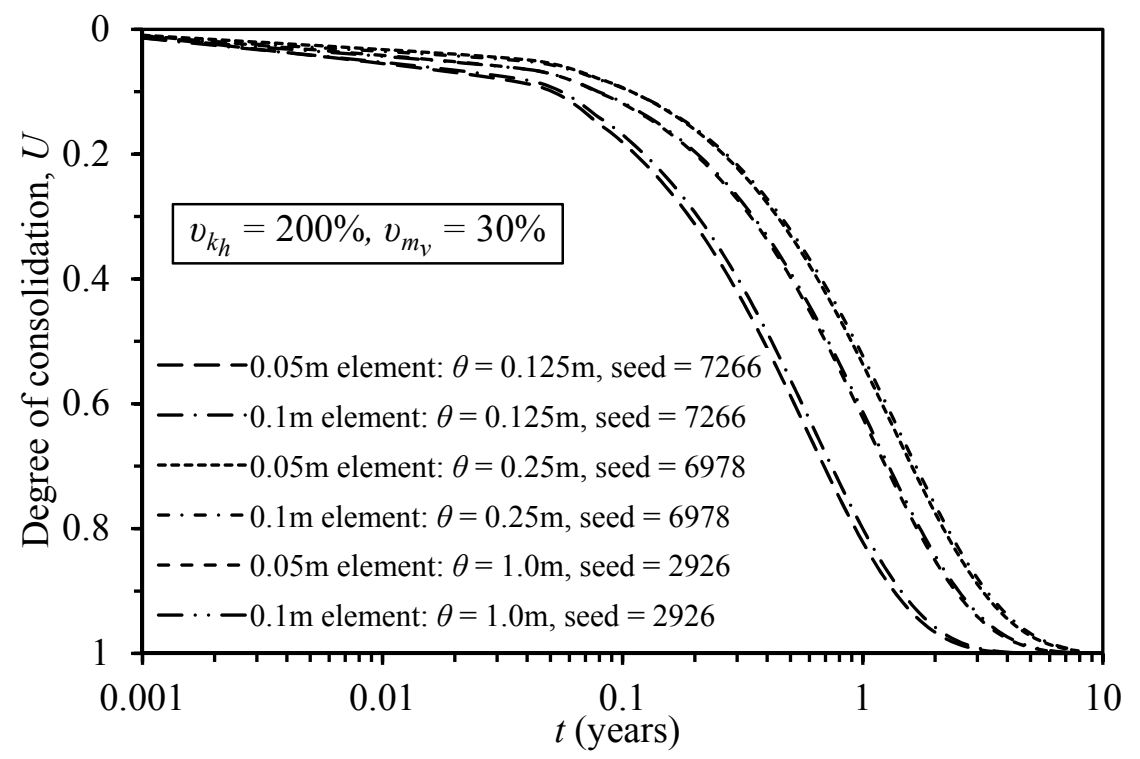

Figure 3. Mesh sensitivity analysis under different scales of fluctuation and seeds. 


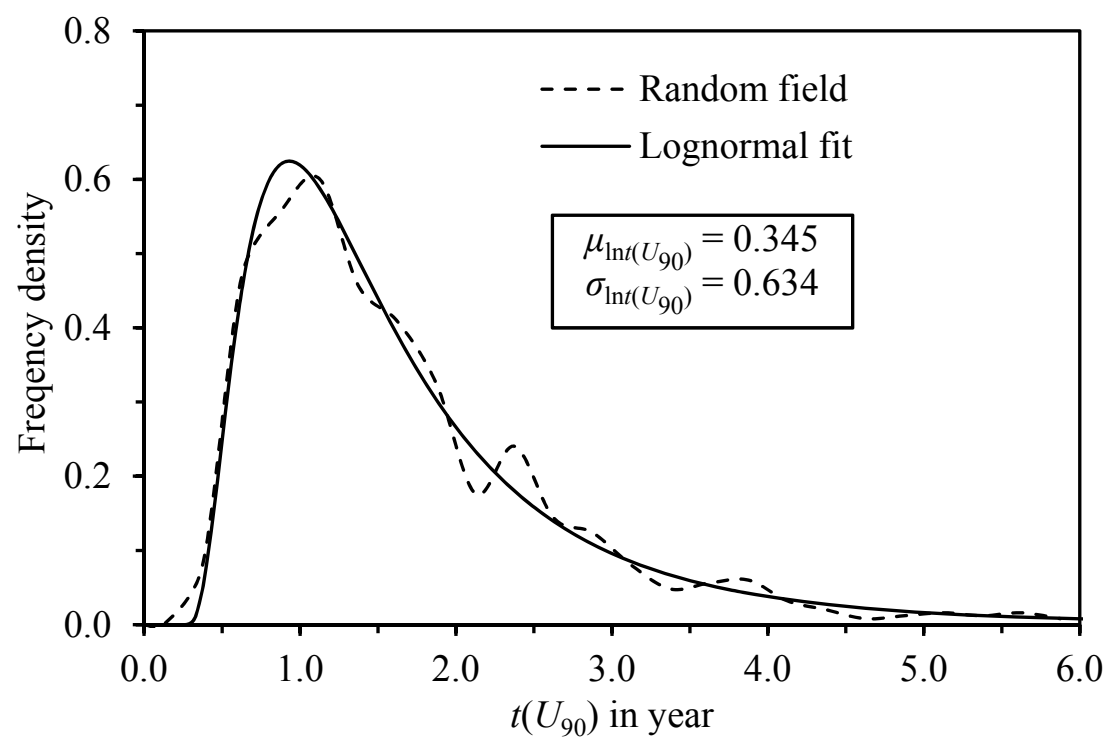

Figure 4. Typical example of frequency density histogram of simulated $t\left(U_{90}\right)$ with fitted lognormal distribution for $v_{k_{u}}=50 \%, v_{k_{s}}=200 \%, v_{m_{v_{u}}}=10 \%, v_{m_{v_{s}}}=30 \%$,

$$
\theta_{k_{u}}=\theta_{k_{s}}=\theta_{m_{v_{u}}}=\theta_{m_{v_{s}}}=0.5 \mathrm{~m}
$$



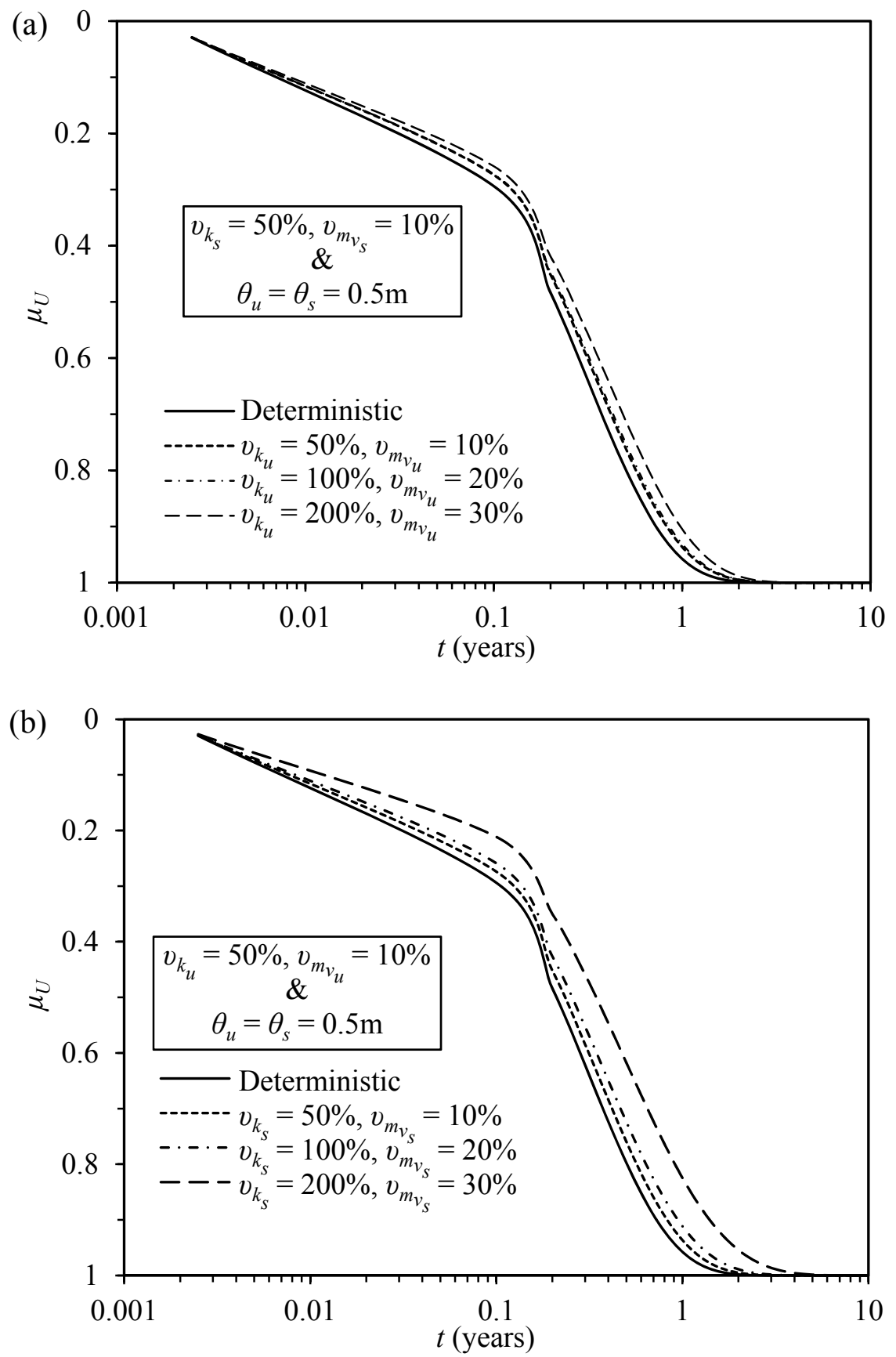

Figure 5. Effect of: (a) $v_{u}$ and; (b) $v_{s}$ on $\mu_{U}$ for $\theta_{u}=\theta_{s}=0.5 \mathrm{~m}$. 

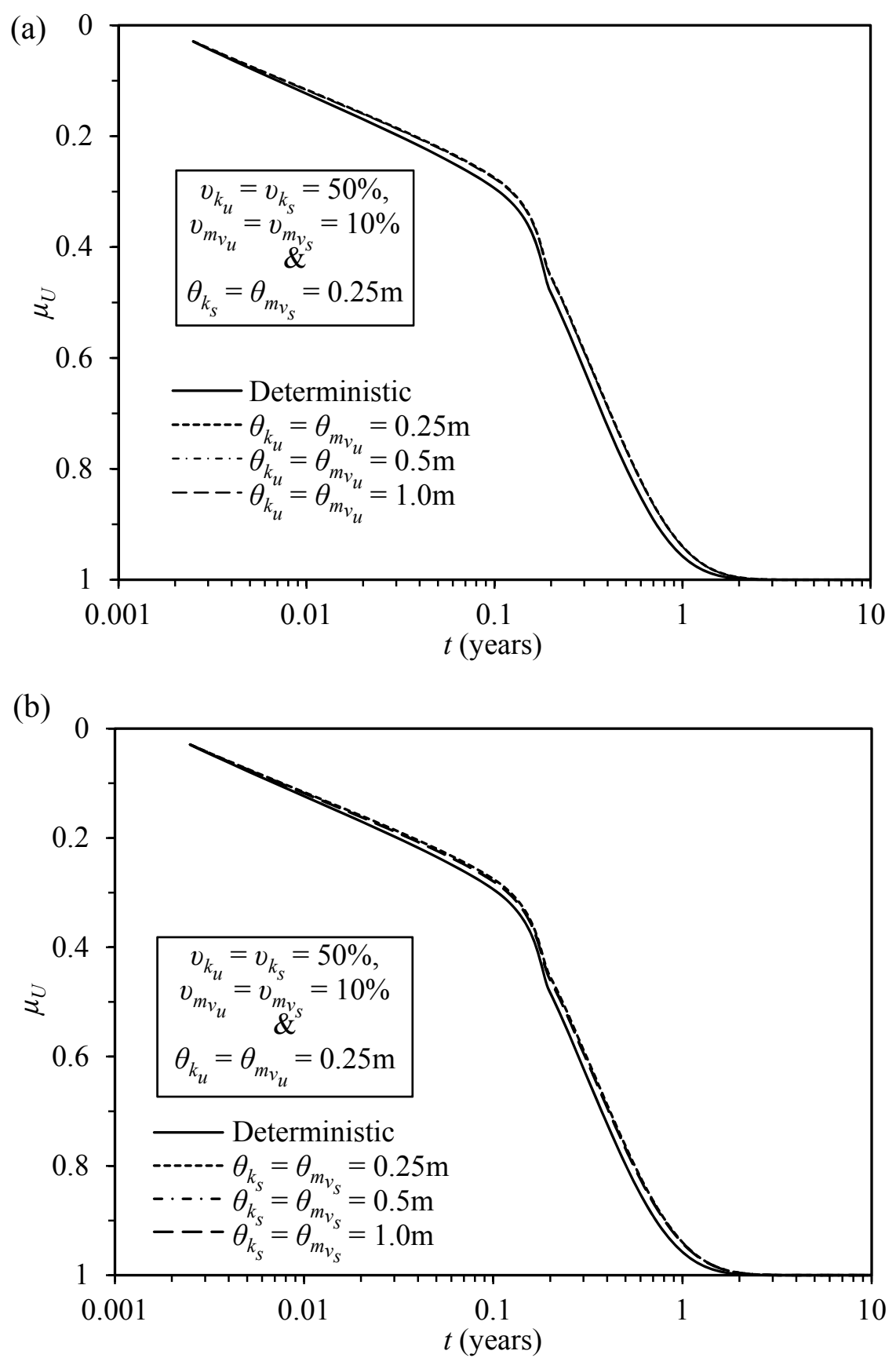

Figure 6. Effect of: (a) $\theta_{u}$ and; (b) $\theta_{s}$ on $\mu_{U}$ for $v_{k_{u}}=v_{k_{s}}=50 \%$ and $v_{m_{v_{u}}}=v_{m_{v_{s}}}=10 \%$. 

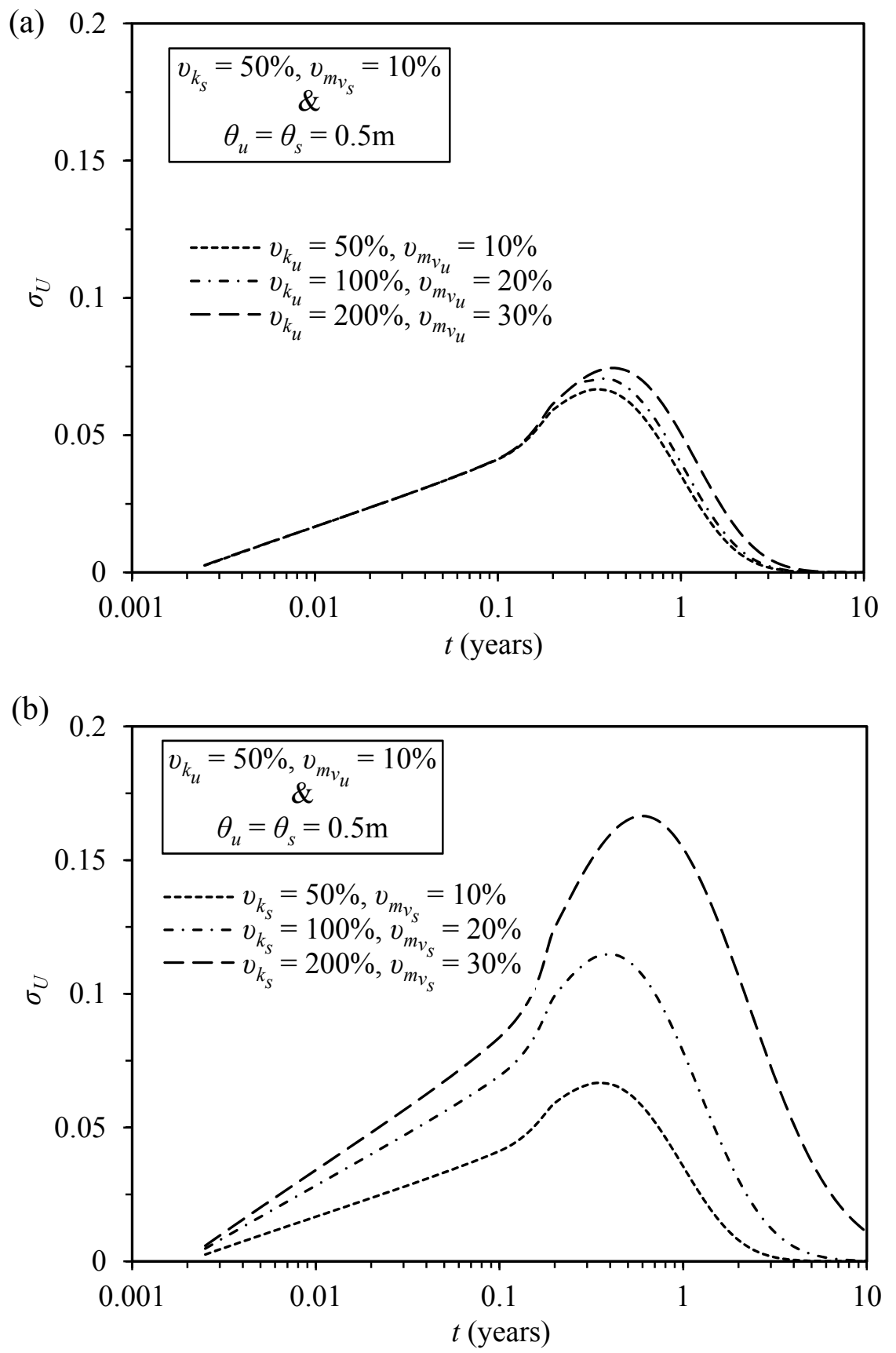

Figure 7. Effect of: (a) $v_{u}$ and; (b) $v_{s}$ on $\sigma_{U}$ for $\theta_{u}=\theta_{s}=0.5 \mathrm{~m}$. 

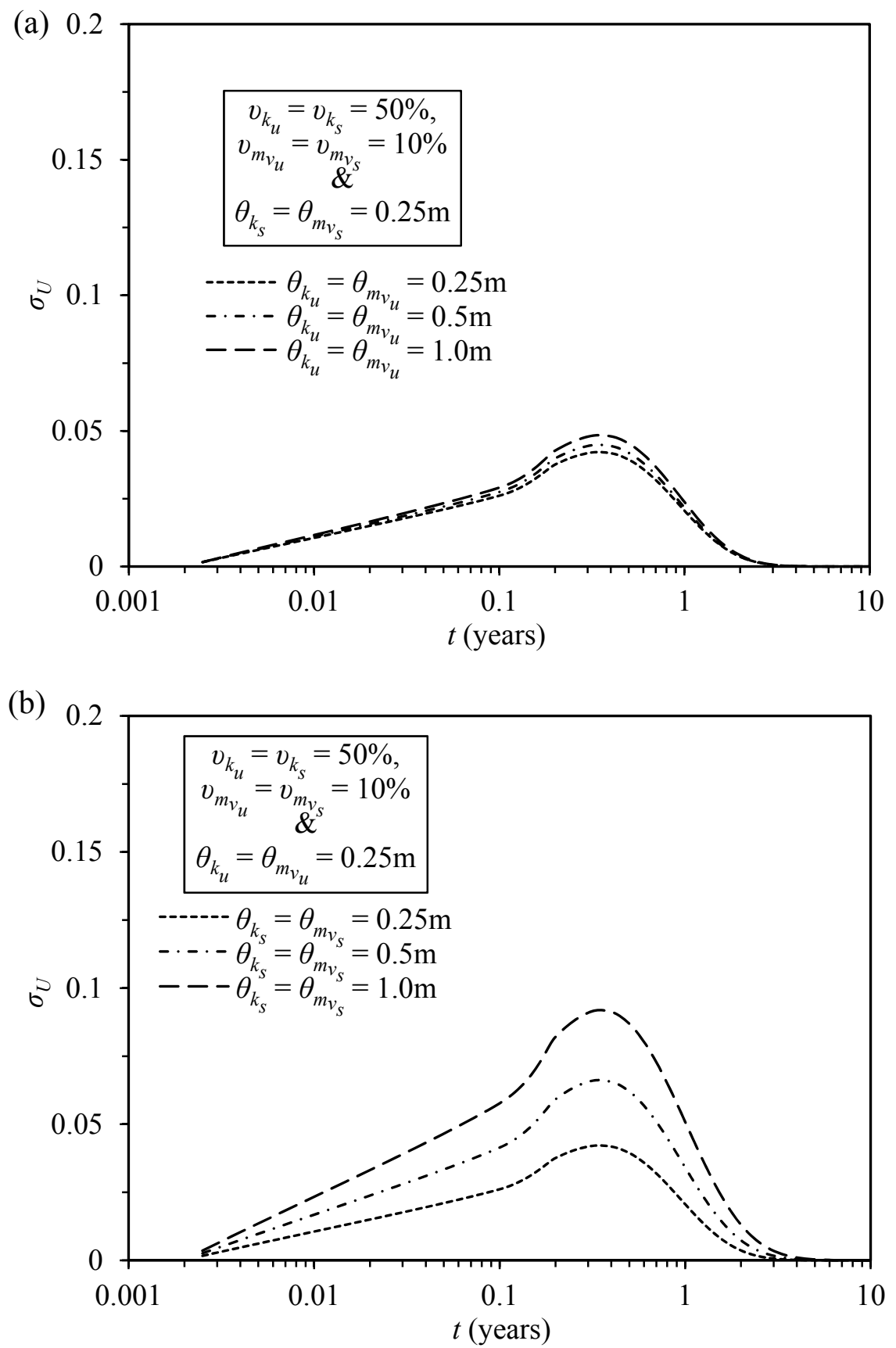

Figure 8. Effect of: (a) $\theta_{u}$ and; (b) $\theta_{s}$ on $\sigma_{U}$ for $v_{k_{u}}=v_{k_{s}}=50 \%$ and $v_{m_{v_{u}}}=v_{m_{v_{S}}}=10 \%$. 

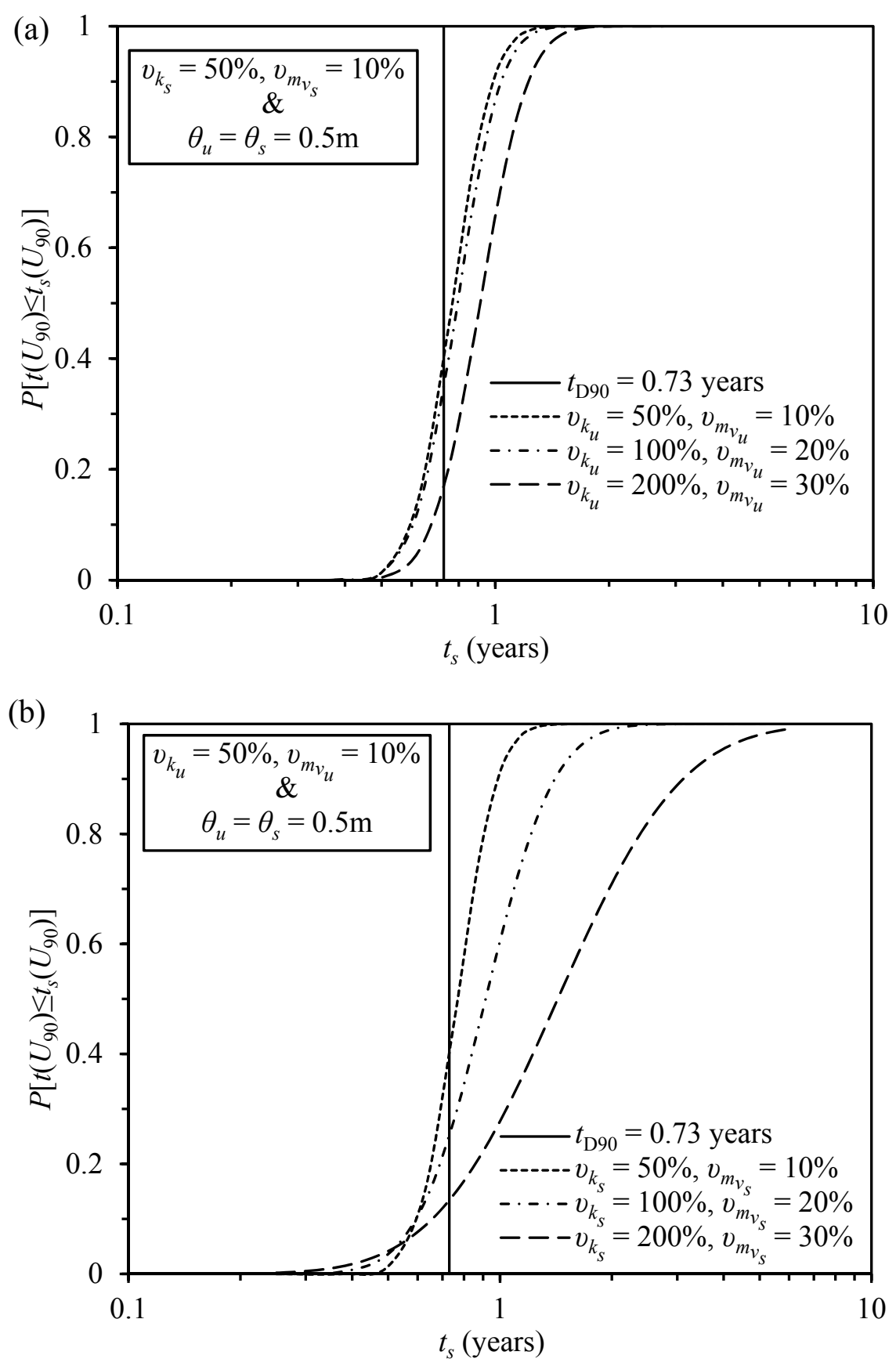

Figure 9. Effect of: (a) $v_{u}$ and; (b) $v_{s}$ on $P\left[t\left(U_{90}\right) \leq t_{s}\left(U_{90}\right)\right]$ for $\theta_{u}=\theta_{s}=0.5 \mathrm{~m}$. 

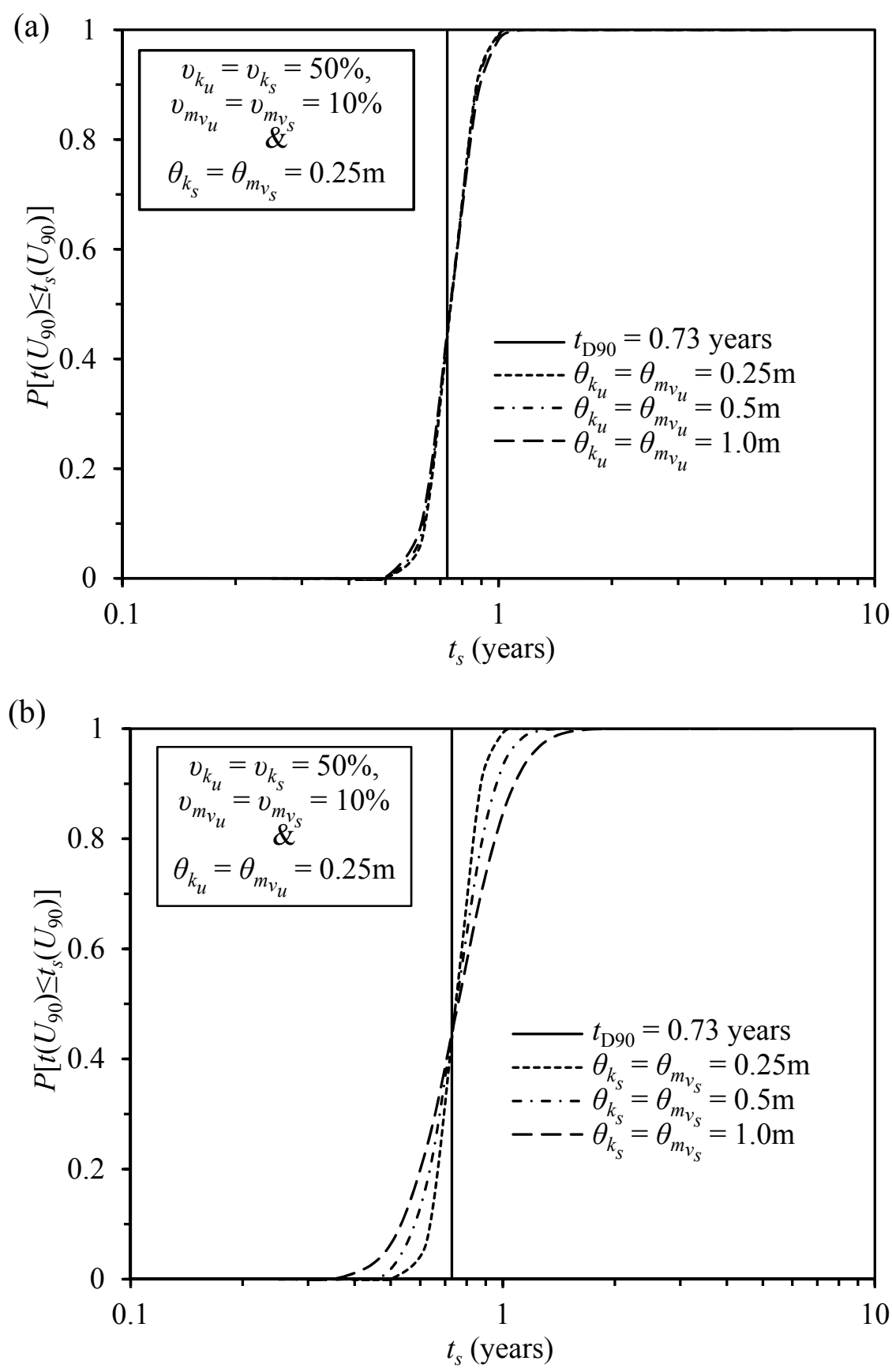

Figure 10. Effect of: (a) $\theta_{u}$ and; (b) $\theta_{s}$ on $P\left[t\left(U_{90}\right) \leq t_{s}\left(U_{90}\right)\right]$ for $v_{k_{u}}=v_{k_{s}}=50 \%$ and

$$
v_{m_{v_{u}}}=v_{m_{v_{s}}}=10 \% .
$$



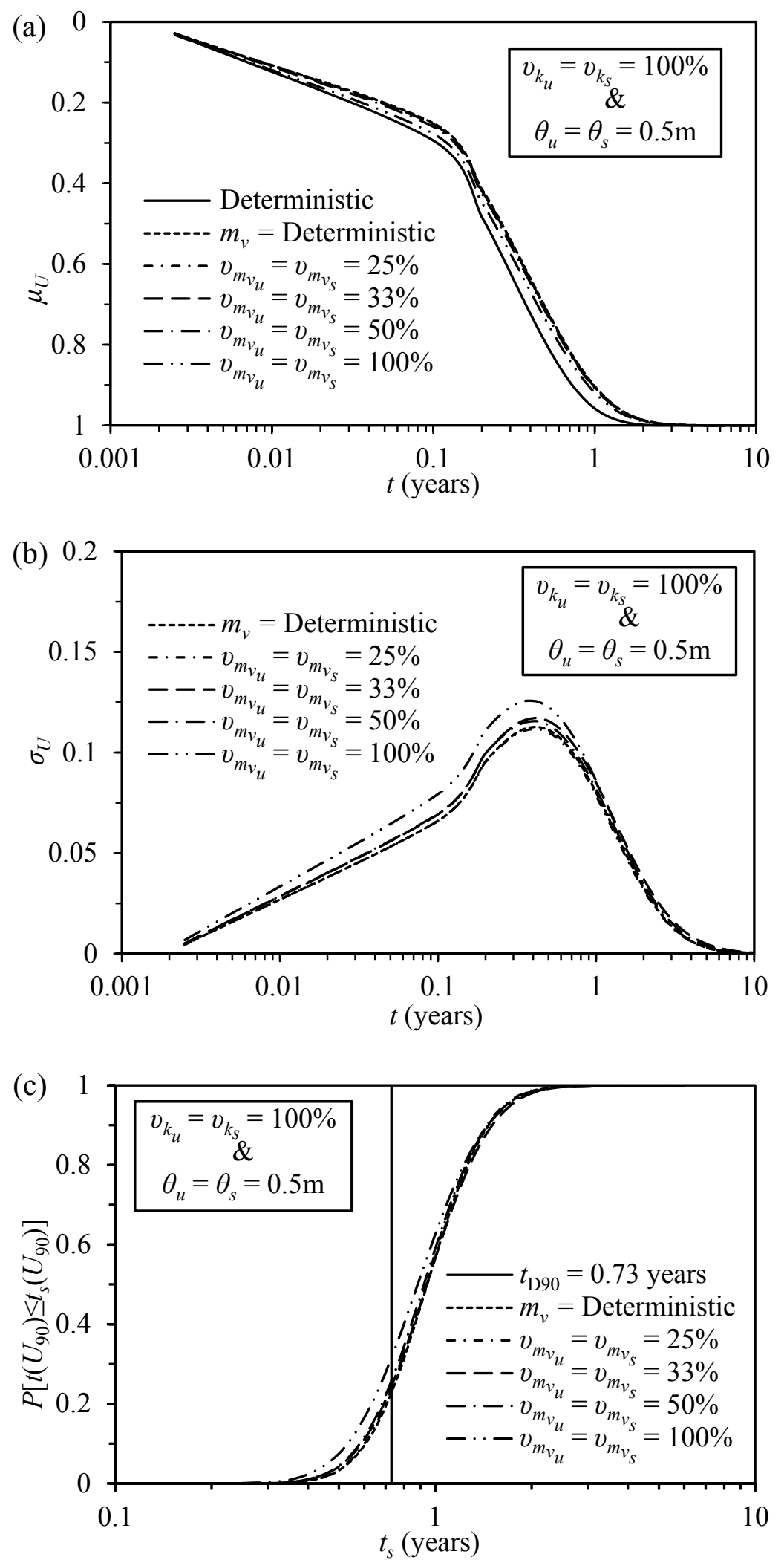

Figure 11. Effect of degree of variability of $m_{v}$ on (a) $\mu_{U}$ (b) $\sigma_{U}$ and (c) $P\left[t\left(U_{90}\right) \leq\right.$ $\left.t_{s}\left(U_{90}\right)\right]$ for $\theta_{u}=\theta_{s}=0.5 \mathrm{~m}$. 